\title{
Cidades seguras e resilientes? Das metas de desenvolvimento sustentável à mortalidade evitável em pequenos municípios
}

A Agenda 2030 para o Desenvolvimento Sustentável direciona metas que perpassam o tripé do desenvolvimento sustentável, entre as metas está a redução da violência e o aumento da segurança rodoviária. O presente estudo tem por objetivo analisar o potencial para o alcance das metas de desenvolvimento sustentável da agenda de 2030, a partir do cenário da mortalidade por causas externas evitáveis. Foi realizado um estudo ecológico, com os coeficientes de mortalidade por causas externas, na população jovem dos municípios da 16a Coordenadoria Regional da Saúde do Rio Grande do Sul, em 2014. Foram utilizados os coeficientes brutos e ajustados pelo método bayesiano empírico local, de mortalidade por causas externas e realizada a distribuição e análise espacial, através do cálculo do Índice de Moran. A correlação de Pearson possibilitou avaliar a relação entre os coeficientes de mortalidade com as variáveis: taxa de analfabetismo na população com idade $=15$ anos, percentual de população com idade $=15$ anos residente na zona urbana, renda média domiciliar per capita, taxa de desemprego na população com idade $=16$ anos e Produto Interno Bruto (PIB) per capita. O teste G foi utilizado para verificar a probabilidade entre o sexo e as variáveis faixa etária, cor e escolaridade das vítimas. As principais causas de mortalidade em jovens foram as agressões (40,3\%) e os acidentes de transporte (37,3\%). A implantação ou implementação de políticas públicas relacionadas é necessária para o alcance das metas de desenvolvimento sustentável pactuados na agenda de 2030 , uma vez que a tendência para a mortalidade por causas evitáveis, como as agressões e os acidentes de trânsito, é superior à mortalidade por doenças crônicas.

Palavras-chave: Indicadores de Desenvolvimento Sustentável; Territórios Sustentáveis; Violência; Agressão; Acidentes de Transporte.

\section{Safe and resilient cities? From sustainable development goals to preventable mortality in small municipalities}

\begin{abstract}
The 2030 Agenda for Sustainable Development targets goals that go beyond the tripod of sustainable development, among the goals is the reduction of violence and the increase of road safety. This study aims to analyze the potential for achieving the sustainable development goals of the 2030 agenda, based on the scenario of mortality from preventable external causes. An ecological study was carried out, with the coefficients of mortality from external causes, in the young population of the municipalities of the 16th Regional Coordination of Health of Rio Grande do Sul, in 2014. The coefficients used and adjusted by the local empirical Bayesian method of mortality were used. by external causes and the spatial distribution and analysis was carried out by calculating the Moran Index. Pearson's correlation made it possible to assess the relationship between mortality coefficients and the variables: illiteracy rate in the population aged $=15$ years, percentage of population aged $=15$ years living in the urban area, average household income per capita, unemployment rate in the population aged $=16$ years and Gross Domestic Product (GDP) per capita. The $\mathrm{G}$ test was used to verify the probability between sex and the victims' age, color and education variables. The main causes of mortality in young people were assaults $(40.3 \%)$ and transport accidents (37.3\%). The implementation or implementation of related public policies is necessary to achieve the sustainable development goals agreed on the 2030 agenda, since the tendency towards mortality from preventable causes, such as aggressions and traffic accidents, is higher than mortality due to chronic diseases.
\end{abstract}

Keywords: Sustainable Development Indicators; Sustainable Territories; Violence; Aggression; Transport Accidents.

Topic: Geodésia, Cartografia e Sensoriamento Remoto

Reviewed anonymously in the process of blind peer.
Received: 02/10/2020

Approved: $\mathbf{2 4 / 1 1 / 2 0 2 0}$
Jéssica Mazutti Penso de Campos (iD Universidade do Vale do Taquari, Brasil http://lattes.cnpq.br/2758147131747627 http://orcid.org/0000-0002-9934-0675 jessica.m.penso@gmail.com

Eliane Fraga da Silveira (D)

Universidade Luterana do Brasil, Brasil http://lattes.cnpq.br/4388826053824317

http://orcid.org/0000-0002-0992-5136 elianefraga3@hotmail.com

\section{Eduardo Périco (iD)}

Universidade do Vale do Taquari, Brasil

http://lattes.cnpq.br/4494244221645524

http://orcid.org/0000-0002-2926-6246

perico@univates.br

\section{Referencing this:}

CAMPOS, J. M. P.; SILVEIRA, E. F.; PÉRICO, E.. Cidades seguras e resilientes? Das metas de desenvolvimento sustentável à mortalidade evitável em pequenos municípios. Revista Ibero Americana de Ciências Ambientais, v.11, n.6, p.274-283, 2020. DOI: http://doi.org/10.6008/CBPC2179-6858.2020.006.0023

DOI: 10.6008/CBPC2179-6858.2020.006.0023 


\section{INTRODUÇÃO}

O relatório Transforming our World: The 2030 Agenda for Sustainable Development foi considerado o documento final da Conferência das Nações Unidas sobre Desenvolvimento Sustentável (Rio+20). Apresenta, em seu contexto, uma declaração que contempla 17 objetivos e 169 metas, que perpassam diferentes temáticas das três dimensões do desenvolvimento sustentável, a social, a econômica e a ambiental (UN, 2015).

O documento enfatiza a importância das ações a nível local, mesmo diante da natureza global da declaração. A paz, a redução da violência e a segurança rodoviária estão entre algumas das metas, e reiteram a importância de uma sociedade mais pacífica e humanizada, a fim de caminhar rumo à sustentabilidade (UN, 2015). No Brasil estudos apontam para o grande problema da mortalidade por violência (NEVES et al., 2015; REICHENHEIM et al., 2011; MOURA et al., 2015) e por acidentes de trânsito (NEVES et al., 2015; MARTINS et al., 2013; MORAIS NETO et al., 2012), caracterizando um grupo de causas externas e evitáveis de óbitos, trazendo a necessidade de reavaliação das ações vigentes, a fim de possibilitar que as metas de desenvolvimento sustentável, relacionadas à essa temática, sejam alcançadas.

Um estudo que analisou os óbitos de jovens de 15 a 29 anos de idade, ocorridos no Brasil entre o ano de 2000 e 2012, apontou que, enquanto houve redução na mortalidade associada às doenças infecciosas e parasitárias e nas causas mal definidas, houve aumento da proporção de óbitos de jovens por causas externas em quase todas as grandes regiões do País. Do total dos óbitos de jovens por causas externas, 54,6\% foram por agressões e $25,3 \%$ por acidentes de transporte. Houve diferença em relação ao sexo, sendo que, entre os homens, $57,0 \%$ dos óbitos foram por agressões e $23,7 \%$ por acidentes de transporte, em contrapartida, entre as mulheres jovens, os acidentes de transporte foram a principal causa de óbito por causas externas $(38,8 \%)$, seguidos pelas agressões $(33,9 \%)$ e lesões autoprovocadas intencionalmente $(10,8 \%)$ (NEVES et al., 2015).

A morte prematura e evitável de jovens por homicídio é um fenômeno que tem crescido no Brasil, desde a década de 1980. Especificamente entre 2007 e 2017, essa taxa apresentou aumento de 37,5\% e, mais precisamente, entre 2016 e 2017 o aumento foi de 6,7\%. No ano de 2017, foi registrada a soma de 35.783 óbitos de jovens assassinados no Brasil, representando a taxa de 69,9 homicídios para cada 100 mil jovens no País, taxa recorde nos últimos dez anos, enquanto que a Organização Mundial da Saúde (OMS) já considera como epidêmica a taxa acima de 10 homicídios por 100 mil habitantes.

Outra tendência observada nos últimos anos é o aumento da mortalidade por violência contra públicos específicos, entre eles, os negros, a população LGBTI e as mulheres. Em relação à faixa etária, entre os 15 e os 19 anos de idade os homicídios representaram a causa de $51,8 \%$ dos óbitos, entre os 20 e os 24 anos $49,4 \%$ e entre os 25 e os 29 anos 38,6\% das mortes consideradas evitáveis, por homicídios, no País.

O Brasil observa a mais profunda transição demográfica já vivenciada, com alta tendência para o padrão de envelhecimento da população, concomitante à alta mortalidade de jovens por causas evitáveis relacionadas à violência, que traz consequências para o desenvolvimento sustentável e para o alcance das 
metas pactuadas na Rio+20.

As ações são orientadas no sentido local para o global, a fim de que as realidades, a cultura, os saberes e práticas, assim como as fragilidades e as potencialidades locais sejam consideradas como parte do processo de desenvolvimento sustentável (UN, 2015). O desenvolvimento sustentável é holístico e precisa ser planejado de acordo com essa natureza, levando em consideração as diferentes propriedades que transcorrem as dimensões envolvidas (ELKINGTON, 1997; ELKINGTON, 1994).

Tendo em vista que as questões acerca da paz, da redução da violência e da segurança rodoviária perpassam as metas da agenda de 2030 para o Desenvolvimento Sustentável, o presente estudo tem por objetivo analisar o potencial para o alcance das metas de desenvolvimento sustentável da agenda de 2030 , na perspectiva da violência e da segurança rodoviária, tendo como método de análise a mortalidade por causas externas evitáveis.

\section{MATERIAIS E MÉTODOS}

Foi realizado um estudo ecológico, observacional, de dados agregados humanos. A unidade de análise foi o conjunto dos óbitos por causas externas evitáveis, ocorridos na população jovem, com idade entre 15 e 29 anos. Essa faixa etária foi escolhida visando analisar as principais causas de óbitos, com a mínima interferência do viés que poderia estar relacionado às doenças crônicas e degenerativas, mais associadas à população adulta-idosa.

O espaço geográfico foi delimitado pelos municípios que integram a 16a Coordenadoria Regional da Saúde do estado do Rio Grande do Sul - Brasil (16ạ CRS/RS/BR), e o período de referência de dados foi o ano de 2014. A área espacial foi escolhida por integrar municípios que não fazem parte da região metropolitana do Estado, típicos do interior, na maioria deles de pequeno porte populacional, a fim de controlar fatores de confusão e falácia ecológica. O conjunto de dados coletados no período de um ano, também visou contribuir no controle da falácia ecológica. Em suma, foram considerados todos os óbitos de jovens residentes na 16ạ CRS/RS/BR, no ano de 2014.

\section{Área do estudo}

O estado do Rio Grande do Sul é uma das 27 unidades federativas do Brasil, situado no extremo sul, apresentando limites geográficos com o estado de Santa Catarina ao norte, a Argentina ao oeste, Uruguai ao sul e o Oceano Atlântico ao leste. A população estimada no Estado é de 11.329 .605 habitantes e a área territorial de $281.731,445 \mathrm{~km}^{2}$. O Estado possui 497 municípios, três grandes bacias hidrográficas: Guaíba, Litoral e Uruguai, relevo característico de extensa baixada com um planalto ao norte, o clima é subtropical e a economia fundamentada na agricultura (principalmente com o cultivo de soja, trigo, arroz e milho), pecuária e indústria (especialmente ligada ao couro/calçados, alimentícia, têxtil, madeireira, metalúrgica e química) (IBGE, 2020a).

O Estado contempla dois biomas, Mata Atlântica e Pampa, dos quais derivam distintos ecossistemas, cada qual caracterizado por singularidades relacionadas ao clima, solos e relevo, ou seja, possui uma 
biodiversidade local única com espécies endêmicas. O bioma Mata Atlântica, tipificado pela existência prevalecente de vegetação florestal, estende-se por cerca de $37 \%$ do território estadual, abrangendo a metade norte do território, apesar de possuir 7,5\% de áreas remanescentes, devido à antropização e fragmentação, embora aproximadamente 2.931 .900 ha das áreas remanescentes encontram-se protegidas desde 1993, instituindo-se a Reserva da Biosfera da Mata Atlântica do Rio Grande do Sul (IBGE, 2020b).

O Bioma Pampa, que também recebe a designação de Campos do Sul ou Campos Sulinos, restringese nacionalmente ao estado do Rio Grande do Sul, ocupando aproximadamente 63\% do território. Caracteriza-se por um conjunto de vegetação de campo em relevo, predominante de planície, com alto grau de diversidade de fauna e flora (IBGE, 2020b).

O rendimento mensal domiciliar per capita foi estimado em $\mathrm{R} \$ 1.635,00$, com $67,6 \%$ da população com idade igual ou acima aos 16 anos em trabalho formal no ano de 2016, aumentando para 70,9 \% em 2017 e rendimento médio é de $\mathrm{R} \$ 2.427,00$. O Índice de Desenvolvimento Humano (IDH) no ano censitário foi de 0.746 (2010), apontando para um alto patamar e o total de veículos, no ano de 2016, foi de 6.650.259. Em relação ao acesso ao ensino, foram registradas 1.323.307 matrículas no ensino fundamental nas 6.052 escolas e 347.637 matrículas no ensino médio em 1.510 escolas, em 2017 (IBGE, 2020a).

A capital do estado é a cidade de Porto Alegre, com população estimada em 1.479 .101 habitantes no ano de 2018, área da unidade territorial em $496,682 \mathrm{~km}^{2}$ e densidade demográfica $2.837,53 \mathrm{hab} . / \mathrm{km}^{2}$. A capital é a cidade de maior porte populacional, a grande parte $(75 \%)$ dos municípios gaúchos segue a tendência do Brasil no elevado número de municípios de pequeno porte. Os dados apontam que em 2010, $60 \%$ dos municípios brasileiros contavam com população igual ou inferior a 15.000 habitantes (IBGE, 2020a).

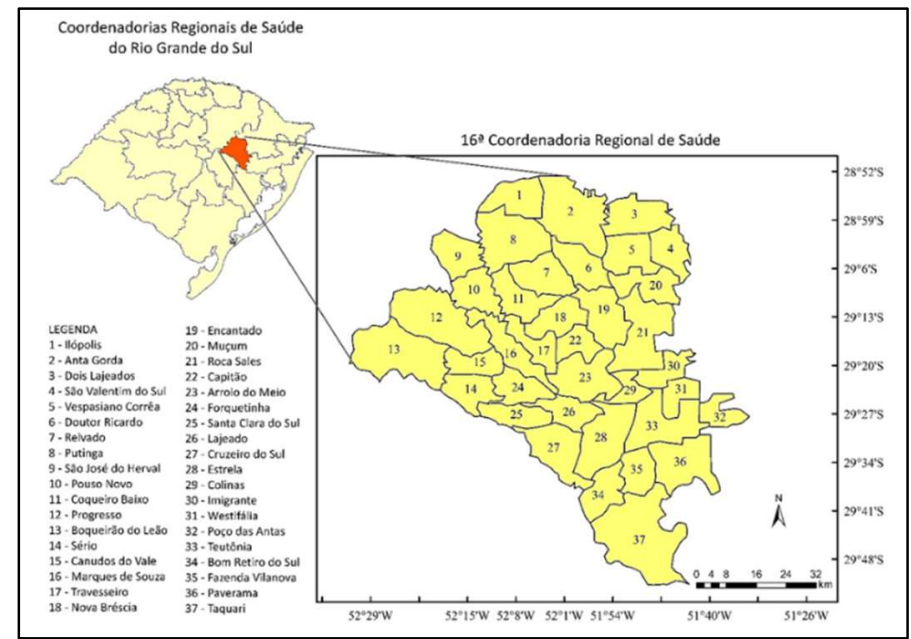

Figura 1: Área geográfica de desenvolvimento do estudo, 16ạ Coordenadoria Regional de Saúde do Rio Grande do Sul, Brasil, 2020.

O Rio Grande do Sul está dividido em 30 regiões de saúde, organizadas por espaço geográfico que integram agrupamentos de municípios, delimitado a partir de identidades culturais, econômicas e sociais e de redes de comunicação e infraestrutura de transportes compartilhados, visando integrar a organização, o planejamento e a execução de ações e serviços de saúde (BRASIL, 2011). 16ª CRS/RS/BR está localizada na bacia do Rio Taquari, na encosta inferior do nordeste do Estado, é composta por duas regiões de saúde, a 
região 29 - Vales e Montanhas e a região 30 - Vale da Luz. A região 29 possui 27 municípios e a região 30 é contemplada por 10 municípios, totalizando 37 municípios e 325.412 habitantes na 16a CRS/RS/BR (BRASIL, 2020; IBGE, 2020a). O município de Lajeado é a sede da regional e contava, no ano de 2010, com 71.445 habitantes. A regional é caracterizada pela grande quantidade de municípios de pequeno porte populacional, indicando a notória dependência externa para as ações de saúde (Medeiros et al., 2012). Dos 37 municípios, $31(83,8 \%)$ possuem menos de 15 mil habitantes e 21 (56,7\% do total) possuem menos de 5 mil habitantes. A principal fonte de renda da maioria dos municípios é a agropecuária e a maior via de circulação é a BR 386 (IBGE, 2020a).

\section{Coleta e análise de dados}

O Brasil possui o Sistema de Informação em Saúde (SIS) do Departamento de Informática do SUS (DATASUS) (BRASIL, 2020), um instrumento relevante no diagnóstico situacional e de importância notória no catalogar das ações de prevenção, promoção e reabilitação, desenvolvidas nos territórios, e no planejamento da alocação e utilização dos recursos financeiros relacionados à saúde (MARIN, 2010). Um dos subsistemas que integra o SIS é o Sistema de Informação sobre Mortalidade (SIM), fonte oriunda dos dados deste estudo, que é alimentado através das informações dos atestados de óbitos.

Foram coletados os quantitativos de óbitos que compõe a amostra do estudo, por capítulo da Classificação Internacional das Doenças 10a Revisão (CID-10), e na sequência, apresentados os números absolutos e as frequências relacionadas às causas externas específicas de óbitos. O coeficiente bruto de mortalidade por causas externas foi calculado para cada município, o numerador foi o número municipal de óbitos e o denominador o número de habitantes do município no mesmo ano, multiplicado por 100.000 habitantes.

Com intuito de controlar o problema de que a 16a CRS/RS/BR é composta majoritariamente por municípios de pequeno porte populacional, levando em consideração que poucos números de casos podem gerar coeficientes altos de mortalidade, em municípios com populações pequenas, optou-se por calcular a taxa bayesiana empírica local para cada município, que possibilita suavizar as flutuações relacionadas às pequenas áreas, através da combinação linear entre a taxa bruta (eventos/população) ponderada por um valor médio, constituída no método bayesiano empírico local pela média dos municípios vizinhos (BRASIL, 2007).

O coeficiente bruto de mortalidade e o coeficiente ajustado pelo método bayesiano empírico local foram utilizados para a distribuição e para a análise espacial da mortalidade por causas externas nos municípios da 16a CRS/RS/BR. Para a análise espacial foi aplicada o cálculo do Índice de Moran Global, que possibilita identificar se a problemática da mortalidade por causas externas está associada ao conjunto de municípios vizinhos, ou seja, se existe a formação de clusters significativos na área analisada. O índice é uma medida de autocorrelação espacial, cuja hipótese nula é de independência espacial. Os resultados do índice são de -1 a 1, sendo que os valores entre 0 e 1 indicam correlação direta e entre 0 e -1 indicam correlação inversa (BRASIL, 2007). 
A Matriz de proximidade espacial escolhida foi a de primeira ordem $W(n \times n)$ e a estratégia de construção da matriz foi a contiguidade, que considera vizinhos os municípios que dividem fronteira adjacente. A validade estatística do Índice de Moran é estabelecida pelo teste de pseudo-significância, do qual são geradas diferentes permutações dos valores dos indicadores, e cada permutação gera um novo arranjo espacial (BRASIL, 2007), foi adotada a significância mediante 99 permutações.

A correlação de Pearson foi utilizada para avaliar a relação entre os coeficientes de mortalidade com as variáveis: taxa de analfabetismo na população com idade $\geq 15$ anos, percentual de população com idade $\geq 15$ anos residente na zona urbana, renda média domiciliar per capita, taxa de desemprego na população com idade $\geq 16$ anos e Produto Interno Bruto (PIB) per capita. Todas as variáveis tiveram como referência os valores municipais no ano de 2012. Foi analisada a relação entre o sexo e as variáveis faixa etária, cor e escolaridade do através do teste $\mathrm{G}$.

Os dados foram manipulados em planilhas eletrônicas Excel e no software TabWin 32, onde ocorreu também o cálculo do coeficiente bruto de mortalidade. $O$ teste $\mathrm{G}$ foi desenvolvido no software BioEstat 5.0 e a correlação de Pearson no software SPSS 20 . O coeficiente ajustado pelo método bayesiano e a análise do Índice de Moran, foi realizado no TerraView 4.2.2 e a elaboração dos mapas no ArcMap 10.3. A presente pesquisa não apresenta implicações éticas, pois utilizou informações secundárias, de acesso público e agrupadas, impossibilitando a identificação dos sujeitos.

\section{RESULTADOS E DISCUSSÃO}

Foram registrados 93 óbitos, a maior parte deles (72\%) contempla o Capítulo XX da CID-10, caracterizando as causas externas de morbidade e mortalidade, que são causas evitáveis de mortes na população jovem, seguida das neoplasias $(7,5 \%)$ e das doenças do aparelho respiratório $(6,5 \%)$ (Tabela 1$)$. Tendo em vista que as causas externas contemplaram o maior conjunto de óbitos na população em estudo, optou-se em identificar, de forma mais detalhada, quais as causas pontuais destas mortes. As agressões $(40,3 \%)$, os acidentes de transporte $(37,3 \%)$ e as lesões autoprovocadas voluntariamente $(13,4 \%)$ foram as principais causas de mortes em jovens na 16a CRS/RS/BR (Tabela 2). A principal causa de mortalidade de jovens na 16a CRS/RS/BR segue a tendência nacional, sendo que as agressões são a principal causa de óbito em jovens no País (NEVES et al., 2015; BRASIL, 2020) e uma das principais nas regiões das Américas (KRUG et al., 2002).

Em nível mundial, a principal causa de óbitos na população jovem, segundo dados da Organização Mundial da Saúde, são os acidentes de transporte (WHO, 2015). No Brasil, os acidentes de transporte caracterizam a principal causa de mortes em mulheres jovens (NEVES et al., 2015). O estudo aponta que a mortalidade de jovens por causas evitáveis, em especial pelas agressões e os acidentes de transporte, precisam ser melhorados na 16a CRS/RS/BR para o cumprimento das metas pactuadas na Rio+20, em relação à implementação de cidades seguras e resilientes.

Os resultados apontam tendência maior de óbitos em jovens do sexo masculino, com faixa etária dos 20 a 29 anos, brancos e com escolaridade entre 8 a 11 anos (Tabela 3). Um estudo corrobora que no Brasil a 
mortalidade de jovens, por causas externas, é mais frequente em homens (NEVES et al., 2015). Em relação à cor, deve-se levar em consideração que a população da 16a CRS/RS/BR, com 15 anos ou mais, é predominantemente branca (IBGE, 2020a).

Tabela 1: Mortalidade por capítulo (CID-10) na população jovem residente na 16ํㅡ Coordenadoria Regional da Saúde, Rio Grande do Sul - Brasil, 2014.

\begin{tabular}{llll}
\hline Capítulo CID-10 & Capítulo & Óbitos & $(\%)$ \\
\hline Algumas doenças infecciosas e parasitárias & $\mathrm{I}$ & 2 & $(2,2)$ \\
Neoplasias & $\mathrm{II}$ & 7 & $(7,5)$ \\
Doenças endócrinas nutricionais e metabólicas & $\mathrm{IV}$ & 3 & $(3,2)$ \\
Transtornos mentais e comportamentais & $\mathrm{V}$ & 2 & $(2,2)$ \\
Doenças do sistema nervosa & $\mathrm{VI}$ & 2 & $(2,2)$ \\
Doenças do aparelho circulatório & $\mathrm{IX}$ & 1 & $(1,1)$ \\
Doenças do aparelho respiratório & $\mathrm{X}$ & 6 & $(6,5)$ \\
Doenças sist. osteomuscular e tec. conjuntivo & $\mathrm{XIII}$ & 3 & $(3,2)$ \\
Causas externas de morbidade e mortalidade & $\mathrm{XX}$ & 67 & $(\mathbf{7 2 , 0 )}$ \\
\hline
\end{tabular}

Fonte: DATASUS (BRASIL, 2020).

Tabela 2: Mortalidade estratificada por causa (CID-10), do Capítulo XX, na população jovem residente na 16ạ Coordenadoria Regional da Saúde, Rio Grande do Sul - Brasil, 2014.

\begin{tabular}{llll}
\hline Causas CID - 10 & Código da causa & Óbitos & $(\%)$ \\
\hline Acidentes de transporte & 104 & 25 & $(\mathbf{3 7 , 3 )}$ \\
Afogamento e submersões acidentais & 106 & 3 & $(4,5)$ \\
Lesões autoprovocadas voluntariamente & 109 & 9 & $(13,4)$ \\
Agressões & 110 & 27 & $(\mathbf{4 0 , 3 )}$ \\
Todas as outras causas externas & 113 & 3 & $(4,5)$ \\
\hline
\end{tabular}

Fonte: DATASUS (BRASIL, 2020).

Foi identificada correlação significativa $(r=0,403 ; p<0,05)$ entre os coeficientes de mortalidade por causas externas evitáveis em relação à taxa de analfabetismo nos municípios, indicando uma tendência maior de óbitos em municípios com maiores taxas de analfabetismo. Os resultados apontam correlação significativa inversa $(r=-0,332 ; p<0,05)$, entre a mortalidade e renda média domiciliar, apontando que existe uma tendência maior nos coeficientes de mortalidade em municípios onde a renda média domiciliar per capita é menor (Tabela 4). Os jovens estão mais vulneráveis, às causas externas, quando inseridos em um ambiente que possua indicadores desfavoráveis de escolaridade e renda.

Existe um preconceito, no que tange a associação entre a população juvenil vulnerável economicamente, em relação a índices mais elevados de atos violentos. Isso desencadeia uma dificuldade, no acesso destes jovens, ao mercado de trabalho e ao acesso à educação, podendo acarretar prejuízos acerca na inserção social e desenvolvimento econômico destes sujeitos (RIZZINI et al., 2016). Uma pesquisa apontou que, bons indicadores sociais têm potencial para contribuir positivamente no que diz respeito à redução dos homicídios (ANDRADE et al., 2016).

O desenvolvimento sustentável não pode ser encarado como unilateral, visando melhorar apenas algum aspecto do processo, que pareça estar fragilizado. Ele se constitui por um processo holístico, que leva em consideração o tripé social-econômico-ambiental. É evidente, segundo os resultados, que a vulnerabilidade econômica e a baixa escolarização estão associados à mortalidade evitável de jovens, e a implantação de políticas públicas norteadas em um modelo de desenvolvimento, embasado no tripé da sustentabilidade, pode ser um caminho para a modificação desse cenário, visando à redução da mortalidade 
evitável, o aumento da segurança, da humanização e da resiliência, conforme pactuado na Conferência Rio+20.

Tabela 3: Mortalidade por causas externas por sexo em relação à faixa etária, cor e escolaridade da população jovem, residente na 16a Coordenadoria Regional da Saúde, Rio Grande do Sul - Brasil, 2014.

\begin{tabular}{|c|c|c|c|c|}
\hline \multirow{2}{*}{ Variável } & \multicolumn{2}{|c|}{ Masculino } & \multicolumn{2}{|c|}{ Feminino } \\
\hline & $\mathbf{N}$ & (\%) & $\mathbf{N}$ & (\%) \\
\hline \multicolumn{5}{|l|}{ Faixa etária } \\
\hline 15 a 19 anos & 14 & $(20,9)$ & 2 & $(3,0)$ \\
\hline 20 a 24 anos & 22 & $(32,8)$ & 4 & $(6,0)$ \\
\hline 25 a 29 anos & 22 & $(32,8)$ & 3 & $(4,5)$ \\
\hline$p$ & 0,93 & & & \\
\hline \multicolumn{5}{|l|}{ Cor } \\
\hline Branca & 51 & $(77,3)$ & 7 & $(10,6)$ \\
\hline Preta & 2 & $(3,0)$ & 0 & $(0,0)$ \\
\hline Parda & 4 & $(6,1)$ & 2 & $(3,0)$ \\
\hline$p$ & 0,47 & & & \\
\hline \multicolumn{5}{|l|}{ Escolaridade } \\
\hline 1 a 3 anos & 2 & $(25,0)$ & 0 & $(0,0)$ \\
\hline 4 a 7 anos & 2 & $(25,0)$ & 0 & $(0,0)$ \\
\hline 8 a 11 anos & 4 & $(50,0)$ & 0 & $(0,0)$ \\
\hline$p$ & 1,0 & & & \\
\hline
\end{tabular}

Legenda: o teste aplicado foi o teste G. $p$ = probabilidade. Gráfico elaborado segundo dados do DATASUS (BRASIL, 2020).

Tabela 4: Correlação entre o coeficiente de mortalidade por causas externas na população jovem com as variáveis socioeconômicas, na 16a Coordenadoria Regional da Saúde, Rio Grande do Sul - Brasil, 2014.

\begin{tabular}{ll}
\hline Variável & Coeficiente de mortalidade \\
\hline Taxa de analfabetismo & $0,403^{*}$ \\
Renda média domiciliar per capita & $-0,332^{*}$ \\
Taxa de desemprego & 0,058 \\
PIB per capita & $-0,035$ \\
Percentual de população urbana & 0,000 \\
\hline
\end{tabular}

Legenda: ${ }^{*} p<0,05$.

A distribuição e a análise espacial possibilitam compreender o padrão geográfico da mortalidade de jovens, por causas externas, nos municípios da 16ạ CRS/RS/BR. Na análise dos coeficientes brutos, foi observada uma tendência aleatória nos municípios que apresentam coeficientes elevados, essa tendência é confirmada pela estatística de Moran, que apontou valores próximos a zero e não significativos (I= -0,09; $p=1,17$ ) (Figura 1). Porém, conforme descrito anteriormente, a regional possui majoritariamente municípios de pequeno porte populacional, onde poucos casos podem apresentar falsamente coeficientes altos de mortalidade. Para controlar esse problema, foi aplicado o modelo bayesiano empírico.

Os coeficientes ajustados por este modelo apontam para correlação espacial positiva e significativa $(I=0,52 ; p=0,01)$, com tendência de cluster de municípios, com altas taxas de mortalidade evitável, integrando as partes noroeste e central da 16ạ CRS/RS/BR (Figura 1). Os municípios que necessitam de implantação ou implementação de políticas públicas mais urgentes são especialmente: São José do Herval $(0,04)$, Marques de Souza (0,03), Forquetinha $(0,03)$, Arroio do Meio $(0,03)$, Capitão $(0,03)$, Pouso Novo $(0,03)$ e Relvado $(0,03)$.

Analisar os locais mais vulneráveis aos eventos de morbimortalidade é relevante para compreender a situação de saúde de determinado território geográfico, facilitando o planejamento de ações de intervenção. Diante disso, a notória importância de integrar aos estudos epidemiológicos a abordagem espacial, que torna mais visível e representativa as situações e condições de saúde das populações (BRASIL, 
2007a; BRASIL, 2007b; BRASIL, 2006).

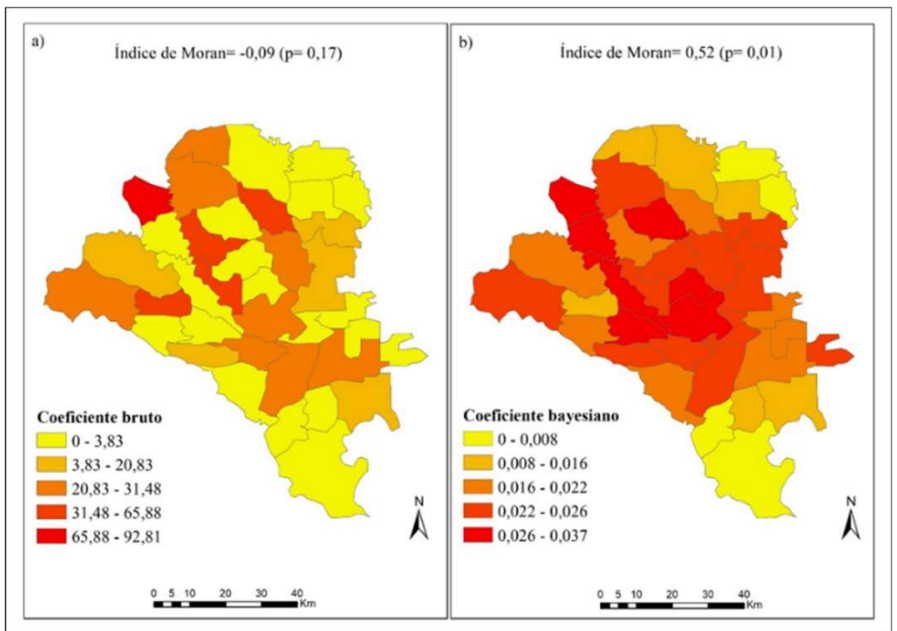

Figura 2: Distribuição espacial dos coeficientes brutos (a) e ajustados (b), de mortalidade por causas externas na população jovem (por 100.000 habitantes), na 16ª Coordenadoria Regional da Saúde, Rio Grande do Sul - Brasil, 2014.

O desenvolvimento sustentável depende de um devir nas ações de pacificação e de conscientização, contribuindo para a cooperação e para o respeito mútuo. Nesta perspectiva, a Agenda 2030 para o Desenvolvimento Sustentável aponta para a importância de ações que possibilitem contribuir com a segurança rodoviária e a redução da violência, em suas diversas formas, tanto em nível global quanto regional ou local. Além disso, enfatizam a importância de monitorar a evolução dos indicadores, acerca das metas, que fazem parte dos objetivos da agenda (UN, 2015).

\section{CONCLUSÕES}

O estudo possibilitou analisar o potencial para o alcance das metas de desenvolvimento sustentável da agenda de 2030, a partir do cenário da mortalidade por causas externas, apontando que a mortalidade por causas evitáveis, em especial pelas agressões e os acidentes de transporte precisam ser melhorados para o cumprimento das metas pactuadas na Rio+20, em relação à implementação de cidades seguras e resilientes.

O maior percentual de óbitos em jovens foi em consequência de causas externas evitáveis, principalmente pelas as agressões, seguida dos acidentes de transporte, que aponta para uma tendência nacional. $\mathrm{O}$ estudo indica quais são os fatores associados e característicos da população mais vulnerável à mortalidade evitável por causas externas e os municípios com indicadores mais alarmantes. Entre as limitações do estudo, está a utilização de dados secundários, que pode apresentar subnotificação, embora o SIM seja um dos subsistemas mais confiáveis do SIS.

Este estudo serve como um importante instrumento para ser duplicado, visando analisar o potencial de desenvolvimento sustentável local-global e o alcance de metas da agenda 2030. Esse é o primeiro passo para avaliar a necessidade de ações de enfrentamento às necessidades e fragilidades relacionadas ao desenvolvimento sustentável, como por exemplo, a implantação ou implementação de políticas públicas relacionadas, a fim de tornar as cidades mais seguras, resilientes e sustentáveis. 
AGRADECIMENTOS: O presente trabalho foi realizado com apoio da Coordenação de Aperfeiçoamento de Pessoal de Nível Superior - Brasil (CAPES) - Código de Financiamento 001, mediante bolsa à primeira autora, e do Conselho Nacional de Desenvolvimento Científico e Tecnológico (CNPq), através de bolsa para EP (processo: 307303/2019-5).

\section{REFERÊNCIAS}

ANDRADE, L.; BRAGA FILHO, H.. A interiorização da violência social: uma análise do interior paulista. Desenvolvimento Regional em debate, v.6, n.1, p.192-217, 2016. DOI: https://doi.org/10.24302/drd.v6i1.934

BRASIL. Decreto n. 7.508, de 28 de junho de 2011. Regulamenta a Lei n. 8.080, de 19 de setembro de 1990 para dispor sobre a organização do Sistema Único de Saúde SUS, o planejamento da saúde, a assistência à saúde e a articulação interfederativa, e dá outras providências. Brasília: DOU, 2011.

BRASIL. Ministério da Saúde. Departamento de Informática do Sistema Único de Saúde (DATASUS). Informações em Saúde. Brasília: MS, 2020.

BRASIL. Ministério da Saúde. Secretaria de Vigilância em Saúde. Fundação Oswaldo Cruz. Abordagens espaciais na saúde pública. Brasília: MS, 2006.

BRASIL. Ministério da Saúde. Secretaria de Vigilância em Saúde. Fundação Oswaldo Cruz. Introdução à Estatística Espacial para a Saúde Pública. Brasília: MS, 2007a.

BRASIL. Ministério da Saúde. Secretaria de Vigilância em Saúde. Fundação Oswaldo Cruz. Sistemas de Informações Geográficas e Análise Espacial na Saúde Pública. Brasília: MS, 2007b.

ELKINGTON, J.. Cannibals With Forks: The Triple Bottom Line of 21st Century Business. Oxford: Capstone, 1997.

ELKINGTON, J.. Triple bottom line revolution: reporting for the third millennium. Canberra: CPA, 1994.

IBGE. Instituto Brasileiro de Geografia e Estatística. IBGE estados: Rio Grande do Sul, 2020a.

IBGE. Instituto Brasileiro de Geografia e Estatística. Mapa de Biomas e de Vegetação, 2020b.

KRUG, E. G.; DAHLBERG L. L.; MERCY J. A.; ZWI A. B.; LOZANO R.. World Report on violence and health. Geneva: World Health Organization, 2002.

MARIN, H. F.. Sistemas de informação em saúde: considerações gerais. Journal of Health Informatics, São Paulo, v.2, n.1, p.20-24, 2010.
MARTINS, E. T.; BOING, A. F.; PERES, M. A.. Motorcycle accident mortality time trends in Brazil, 1996-2009. Revista de Saúde Pública, São Paulo, v.47, n.5, p.931-941, 2013. DOI: https://doi.org/10.1590/S0034-8910.2013047004227

MEDEIROS, C. R. G.; MENEGHEL, S. N.; GERHARDT, T. E. Desigualdades na mortalidade por doenças cardiovasculares em pequenos municípios. Ciência e Saúde Coletiva, Rio de Janeiro, v.17, n.11, p.2953-2962, 2012. DOI: https://doi.org/10.1590/S1413-81232012001100012

MORAIS NETO, O. L.; MONTENEGRO, M. M. S.; MONTEIRO, R. A.; SIQUEIRA JÚNIOR, J. B.; SILVA, M. M. A.; LIMA, C. M.; MIRANDA, L. O. M.; MALTA, D. C.; SILVA JUNIOR, J. B.. Mortalidade por acidentes de transporte terrestre no Brasil na última década: tendência e aglomerados de risco. Ciência e Saúde Coletiva, Rio de Janeiro, v.17, n.9, p.2223-2236, 2012. DOI: https://doi.org/10.1590/S141381232012000900002

MOURA, E. C.; GOMES, R.; FALCÃO, M. T. C.; SCHWARZ, E. NEVES, A. C. M.; SANTOS, W.. Gender inequalities in external cause mortality in Brazil, 2010. Ciência e Saúde Coletiva, Rio de Janeiro, v.20, n.3, p.779-788, 2015. DOI: https://doi.org/10.1590/1413-81232015203.11172014

NEVES, A. C. M.; GARCIA, L. P.. Youth mortality in Brazil: profile and trends in the period 2000-2012. Epidemiologia e Serviços de Saúde, Brasília, v.24, n.4, p.595-606, 2015. DOI: https://doi.org/10.5123/S1679-49742015000400002

REICHENHEIM, M. E.; SOUZA, E. R.; MORAES, C. L.; JORGE, M. H. P. M.; SILVA, C. M. F. P.; MINAYO, M. C. S.. Violence and injuries in Brazil: the effect, progress made, and challenges ahead. Lancet, v.377, n.9781, p.1962-75, 2011. DOI: https://doi.org/10.1016/S0140-6736(11)60053-6

RIZZINI, I.; LIMONGI, N. S.. Percepções sobre violência no cotidiano dos jovens. Revista Katálysis, Florianópolis, v.19, n.1, p.33-42, 2016. DOI: https://doi.org/10.1590/141449802016.00100004

UN. United Nations. Transforming our World: the 2030 agenda for sustainable development. New York: United Nations, 2015.

WHO. World Health Organization. Global status report on road safety 2015. Geneva: WHO, 2015.

A CBPC - Companhia Brasileira de Produção Científica (CNPJ: 11.221.422/0001-03) detém os direitos materiais desta publicação. Os direitos referem-se à publicação do trabalho em qualquer parte do mundo, incluindo os direitos às renocães, expansões e disseminac̃oes da contribuiç̃o, bem como outros direitos subsidiários. Todos os trabalhos publicados eletronicamente poderão posteriormente ser publicados em coletâneas impressas sob coordenação da Sustenere Publishing, da Companhia Brasileira de Produção Científica e seus parceiros autorizados. Os (as) autores (as) posteriormente ser publicados em coletâneas impressas sob coordenação da Sustenere Publishing, da Companhia Brasileira de Produção Cientifica e seus parce 